\title{
Merkel cell carcinoma of the thigh: case report and review of the literature
}

This article was published in the following Dove Medical Press journal: OncoTargets and Therapy

\author{
Wen Jiang* \\ Jiali $\mathrm{Xu}^{*}$ \\ Rong Wang \\ Tingting Wang \\ Yongqian Shu \\ Lianke Liu
}

Department of Oncology, the First Affiliated Hospital of Nanjing Medical University, Nanjing 210029, China

*These authors contributed equally to this work
Correspondence: Lianke Liu Department of Oncology, the First Affiliated Hospital of Nanjing Medical University, No. 300 Guangzhou Road, Nanjing, Jiangsu 210029, China Email liulianke@jsph.org.cn
Background: Merkel cell carcinoma (MCC) is a kind of cutaneous neuroendocrine cancer with a poor prognosis. It is characterized by a high rate of recurrence and metastases, including distant metastases and regional nodal metastases. Clinically, MCC often manifests as obvious single painless hard nodules visible in sun irradiation of diameter $<2 \mathrm{~cm}$ and not uncommonly $>2 \mathrm{~cm}$, with rapid growth and metastases, especially lymph node metastases. Due to the aspecific nature of MCC, it is often confused with other skin cancers. Exploring different treatments of MCC is necessary.

Case presentation: The current study describes the case of an 86-year-old retired man, who presented with a $2.5 \times 2.0 \times 1.2 \mathrm{~cm}$ red nodule on the right thigh, which was initially diagnosed as subcutaneous small cell cancer. Upon histological and immunohistochemical analysis, the tumor was consistent with a diagnosis of MCC.

Results: Antiangiogenic therapy combining endostar and apatinib was administered and a partial response achieved after 2.0 months of treatment, and 6.5 months of progression-free survival was achieved. Overall survival was 13.0 months.

Conclusion: We believe that antiangiogenic therapy is an extremely effective treatment for MCC, especially for patients who cannot tolerate chemotherapy and radiotherapy.

Keywords: Merkel cell carcinoma, antiangiogenic therapy, endostar, apatinib

\section{Background}

Merkel cell carcinoma (MCC) is a kind of cutaneous neuroendocrine cancer with a poor prognosis. It is characterized by a high rate of recurrence and metastases, including distant metastases and regional nodal metastases. There has been controversy about the origins of MCC organization, and different scholars have different views. ${ }^{1,2}$ Clinically, MCC often manifests as obvious single painless hard nodules visible in sun irradiation of diameter $<2 \mathrm{~cm}$ and also not uncommonly $>2 \mathrm{~cm}$, with rapid growth and metastases, especially lymph node metastases. ${ }^{3}$ Clinically, MCC is easily confused with other cancers of the skin, such as metastasis of squamous cell carcinoma, melanoma, and other tumors. The positive expression of certain antibodies in immunohistochemical staining is an important diagnostic tool to distinguish MCC from these tumors. At present, the combination of postoperative radiotherapy and chemotherapy is often used, but there is no standard protocol for chemotherapy. Angiogenesis is an important process in tumor development. Inhibiting vascular regeneration can prolong survival and delay progression of disease. In this paper, we report a case of MCC treated with antiangiogenic therapy in our department combined with a literature review, in order to provide a reference for the clinical diagnosis and treatment of MCC. 


\section{Case presentation}

This case concerns an 86-year-old man who used to be a soldier. He found a mass on the right thigh with a $2.5 \times 2.0 \times 1.2 \mathrm{~cm}$ red nodule at the beginning of May 2017 . Surgical resection was performed at the local People's Hospital. The results of pathological examination after surgery in local hospital showed a right thigh subcutaneous small cell malignant tumor of about $2.5 \times 2.0 \times 1.2 \mathrm{~cm}$ (Figure 1). The pathology immunohistochemistry in our hospital indicated $\mathrm{CK}-\mathrm{L}^{+}, \mathrm{Syn}^{++}, \mathrm{CgA}^{+}, \mathrm{CD} 56^{++}$, Ki67 $\mathrm{CK}_{-} \mathrm{H}^{-}, \mathrm{CK}^{-}{ }^{-}, \mathrm{CK} 20^{+}$, TTF-1-, ${ }^{-} \mathrm{CD} 20^{-}, \mathrm{CD}^{-}$, and $\mathrm{S} 100^{-}$, implying MCC. According to the pathology and immunohistochemistry, he was diagnosed with MCC T2N1M0.

In mid-July 2017, multiple red masses appeared in the surgical incision area, of which the largest was hard and about $1.5 \mathrm{~cm}$ in diameter (Figure 2A). A metastatic lymph node was found in the right inguinal region on computed tomography (CT), $\sim 2.2 \mathrm{~cm}$ in diameter without distant metastasis (Figure 3A). Next-generation sequencing from his peripheral blood showed KDM5 $A^{\mathrm{G} 931 \mathrm{D}}$ mutation, which may have been associated with oncogenesis.

Due to his old age, the patient and his family members refused radiotherapy and chemotherapy. As MCC is closely related to neovascularization, antivascular therapy can be an effective treatment. As such, endostar $30 \mathrm{mg}$ was administered intravenously on day $1-4$ as the first cycle from August 2, 2017. After two cycles, the diameter of the major mass in the right thigh had reduced to $1 \mathrm{~cm}$, and the remaining masses gradually subsided (Figure 2A-C). According to the RECIST standard, efficacy reached partial response. Before the third treatment, the masses became protruding, with a recurrent trend (Figure 2D). On $\mathrm{CT}$, there was no change from before (Figure 3B). Therefore, apatinib was added at $250 \mathrm{mg}$ every other day from the third cycle beginning October 22, 2017. After the next three cycles of treatment, the masses were disappearing (Figure 2D-F), while the diameter of the lymph node in the right inguinal region had reduced to $1.3 \mathrm{~cm}$ (Figure 3C). In summary, after nearly 5 months (five cycles) of endostar and apatinib treatment, clinical efficacy had reached a partial response. During treatment, common adverse reactions of endostar and apatinib, such as proteinuria, hypertension, handfoot syndrome, and hematological toxicity, did not appear.

However, from the beginning of the sixth cycle on February 14, 2018, there was a new lesion of $1.2 \mathrm{~cm}$ diameter at the right thigh (Figure $2 \mathrm{G}$ ), which indicated progressive disease. He then continued to receive endostar and apatinib. At the same time, everolimus $5 \mathrm{mg}$ daily and tegafur $50 \mathrm{mg}$ twice daily were administered. However, he stopped taking everolimus due to serious adverse effects of weakness and vomiting after 2 months. Three treatment cycles later, the diameter of the mass had gradually increased to $3 \mathrm{~cm}$ (Figure 2G-I). The latest CT on March 5, 2018 showed no significant changes compared with previously (Figure 3D). The patient was followed up for about 12 months. Finally, he died of infection at the beginning of June 2018. Progression-free survival was 6.5 months and overall survival 13.0 months.

The patient's family provided written informed consent for publication of this report and accompanying images. We confirmed with the institutional review board of the First Affiliated Hospital of Nanjing Medical University that institutional approval was not required to publish this case report.

\section{Discussion}

MCC is a cutaneous neuroendocrine malignancy. The disease occurs mainly in bodily areas exposed to sunlight, such as the face, neck, and limbs, with low incidence and high recurrence. ${ }^{3}$ People of different ages may develop the disease, especially those $>70$ years. ${ }^{4,5}$ Because MCC is relatively rare, it is easily misdiagnosed as other tumors of the skin, which may cause the lower incidence of the disease. Correct understanding and deeper exploration of MCC have become extremely important. The organizational origin of MCC is unclear. In 1978, Tang et al found that MCC may originate from MCs. MCs are
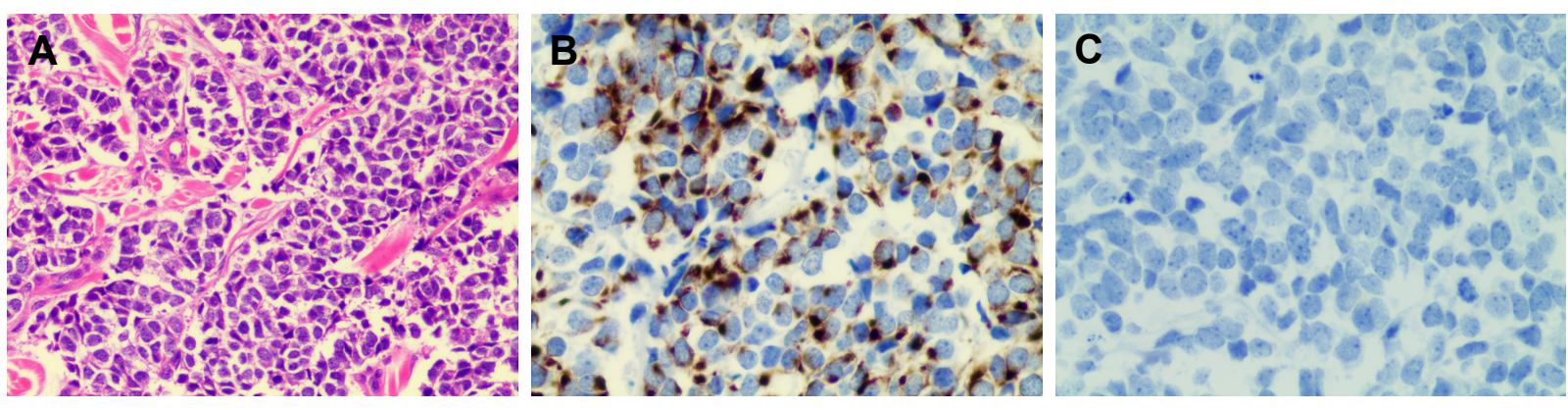

Figure I The pathology and immunohistochemistry of the patient.

Notes: (A) H\&E staining with surgical specimens. (B) Immunohistochemistry: dot-like positivity for CK20. (C) Immunohistochemistry: negativity for CK7. (A) 200×; (B, C) 400×. 
A

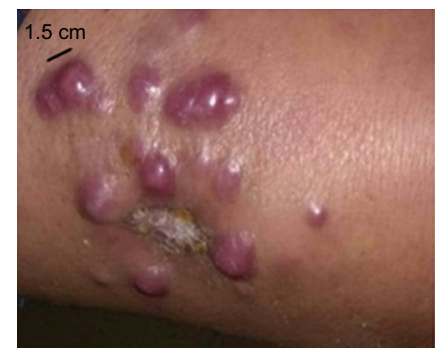

D

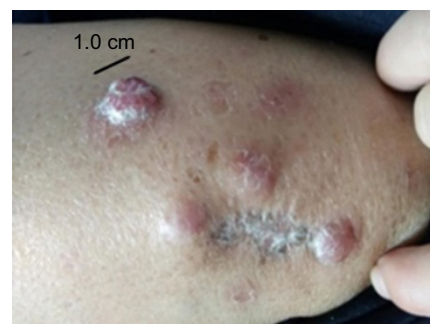

G

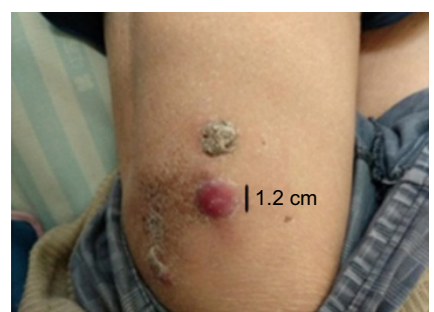

B

E

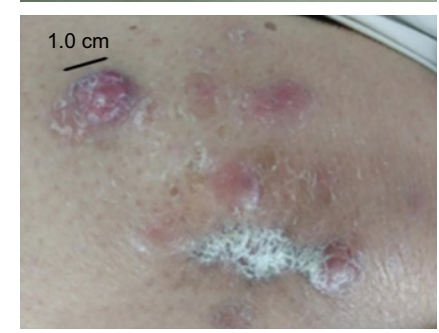

H

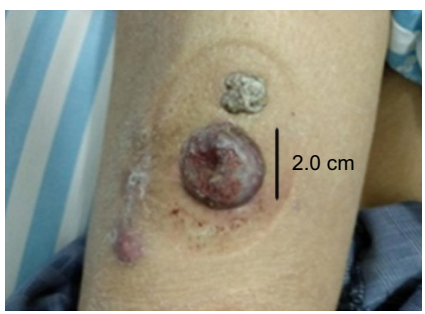

C

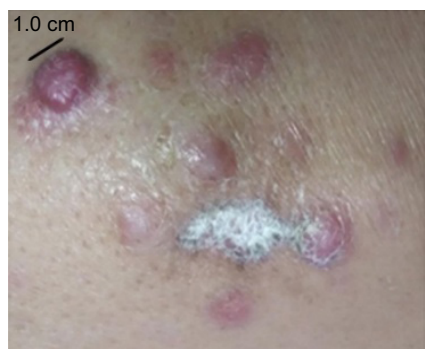

$\mathbf{F}$

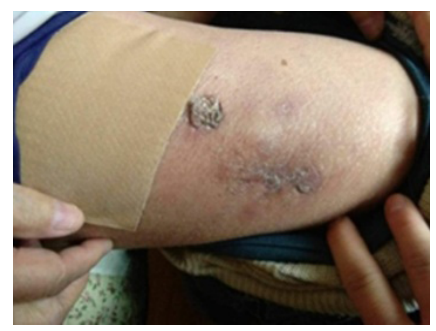

I

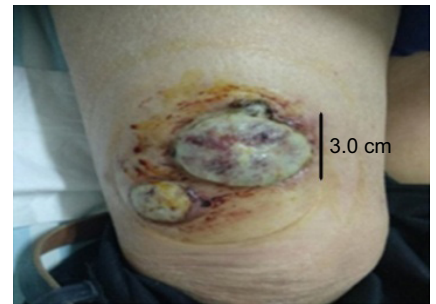

Figure 2 Changes in lesions during treatment of the patient.

Notes: (A) After surgery, before treatment; (B) after the first treatment; (C) after the second treatment; (D) after the third treatment; (E) after the fourth treatment; (F) after the fifth treatment; (G) after the sixth treatment; (H) after the seventh treatment; (I) after the eighth treatment. From the pictures, masses reduced significantly during two cycles $(\mathbf{A}-\mathbf{C})$. From the third cycle $(\mathbf{D})$, tumors had a recurrent trend. After three cycles $(\mathbf{D}-\mathbf{F})$, tumors had started disappearing. From the sixth to eighth cycles $(\mathbf{G}-\mathbf{I})$, the masses were increasing gradually.
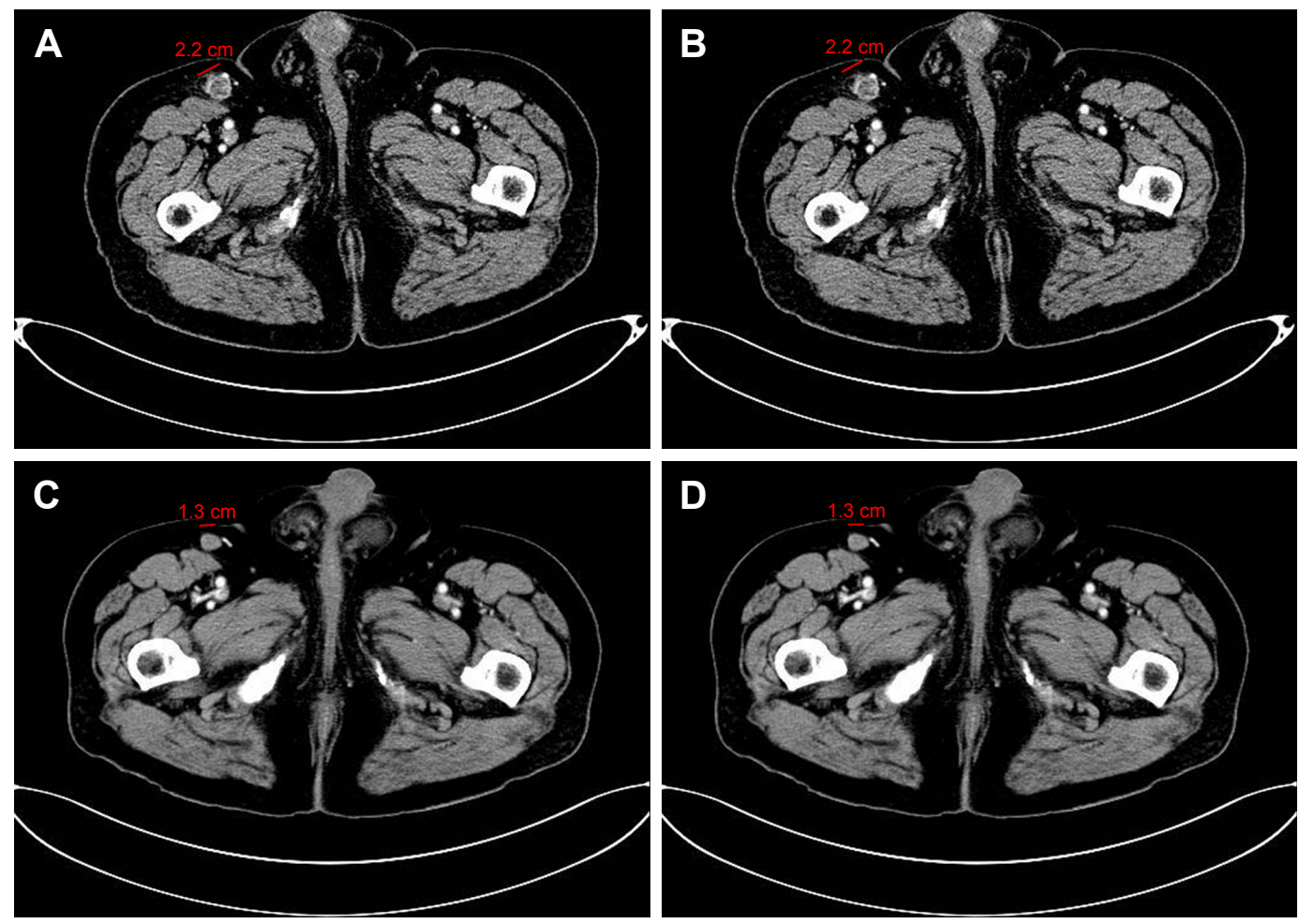

Figure 3 Changes on CT scans during treatment.

Note: (A) Metastatic lymph node $(2.2 \mathrm{~cm})$ on July 31, 2017; (B) metastatic lymph node $(2.2 \mathrm{~cm})$ on October 23,2017 ; (C) metastatic lymph node (I.3 cm) on

December 26, 2017; (D) metastatic lymph node $(1.3 \mathrm{~cm})$ on March 5, 2018.

Abbreviation: $\mathrm{CT}$, computed tomography. 
related to the sense of touch. In lower organisms, this sense of touch is like a mechanical stimulus. ${ }^{1}$ MCs are derived from neuropathies and are members of the amine-precursor-uptake decarboxylation cell system. In recent years, new discoveries and different views on its origin have been gradually emerging. For example, the original multipotent stem cells and some kinds of progenitor cells have become the focus of many scholars, even though the exact basis for the origin of MCC tumors is still lacking. ${ }^{2,6}$ Feng et al found that polyomaviruses could integrate with host genes, with clone proliferation in eight patients with MCC. ${ }^{7}$ This virus had not previously been reported. MC polyomavirus is still considered the causative agent of MCC. Interestingly, polyomaviruses do not actually affect MCs. ${ }^{8} \mathrm{MCC}$ cells are distributed around the epidermis, especially in the skin appendages. According to morphology under microscopy, histological types of MCC are divided into three types: intermediate, small cell, and between the former two. Of these types, the most characteristic is the intermediate type, and the worst prognosis is the small cell type. On the basis of cancer cell morphology, immunohistochemistry can confirm the diagnosis of MCC. It is noteworthy that CK20 is a sensitive and specific indicator of MCC. ${ }^{9} \mathrm{CKs}$ (such as AE1/AE3, CAM5.2, pan-CK), CD5, CD17, CD117, NF, EMA, NSE, TTF1, and $\mathrm{Bcl} 2$, and the epithelial marker BER-EP4 are usually positive in MCC, with the exception of TTF1. The negative expression of TTF1 in combination with the positive expression of CK20 can differentiate MCC and SCLC $\left(\mathrm{TTF}^{+}\right.$and $\left.\mathrm{CK} 20^{-}\right)$to a certain degree. At the same time, $\mathrm{NF}^{+}$combined with $\mathrm{CK} 20^{+}$also contributes to the differential diagnosis of MCC from other neuroendocrine carcinomas. ${ }^{10}$ In this study, $\mathrm{CK} 20^{+}$was found by immunohistochemistry, and related neuroendocrine tumor markers, such as Syn and CD56, were all positive and TTF negative. Compared to other tumors, the incidence of MCC is low. Due to the lack of standard therapy, treatment for MCC is individual. When the patient has a surgical indication, a complete primary tumor resection is needed, and the lymph node is evaluated by sentinel lymph node operation. ${ }^{11,12}$ Adjuvant radiotherapy is recommended for high-risk patients with local recurrence for incomplete resection or larger tumors $(>2 \mathrm{~cm})$. Moreover, Veness's analysis showed that if the simple surgical resection was used, the median survival time was about 6 months. ${ }^{13}$ When postoperative radiotherapy is added, median survival can be extended to 23 months. If lesions have metastasized, such as in lymph-node metastasis and especially distant metastasis, often adjuvant systemic chemotherapy is needed. ${ }^{11}$ Distant metastases usually suggest further postoperative chemotherapy, but a study has reported that combined postoperative chemotherapy may reduce a patient's survival time and increase postoperative recurrence. ${ }^{14}$ Therefore, making an appropriate choice of chemotherapy is crucial. At this point, effective chemotherapy drugs include cisplatin, etoposide, doxorubicin, cyclophosphamide, and vincristine. ${ }^{15-17}$ Although chemotherapy may be effective, a high rate of recurrence leads to a poor prognosis. Recently, some scholars summarized that PD1 or PDL1 inhibition could be regarded as a new first-line therapy for patients with metastatic MCC. ${ }^{18}$ A National Cancer Institute-sponsored clinical trial had an estimated progression-free survival of $67 \%$ at 6 months by studying pembrolizumab (anti-PD1) in 25 patients with advanced MCC. Results eventually showed that pembrolizumab was effective (objective remission rate $62 \%$ ). ${ }^{19}$ In March 2017, avelumab became the first PDL1 monoclonal antibody approved by the US Food and Drug Administration for MCC treatment. In January 2018, National Comprehensive Cancer Network guidelines recommended avelumab, pembrolizumab, and nivolumab as choices for patients with metastatic disease, and these drugs were becoming the new standard for patients with metastatic or unresectable MCC. Endostar and apatinib chemotherapy has not been reported to be used in MCC. In our case report, we recommended the patient receive chemotherapy. Considering the age of the patient, family members refused chemoradiotherapy. The patient could not afford immunotherapy for financial reasons. Finally, individualized treatment with endostar and apatinib was used. Both of these were new biological products for angiogenesis inhibition, whose mechanism was inhibiting the migration of vascular endothelial cells, leading to an inhibition of tumor angiogenesis, blocking the nutritional supply of tumor cells, as well as achieving the purpose of inhibiting tumor proliferation or metastasis. Endostar can suppress the proliferation and migration of endothelial cells by inhibiting VEGFR signaling, repressing the cell cycle, and controlling antiapoptosis genes, eventually resulting in cell death. ${ }^{20,21}$ In a clinical trial of breast cancer, 803 patients who were treated with neoadjuvant docetaxel, epirubicin, and endostar showed a relatively higher pathological complete response than the patients treated with just docetaxel + epirubicin. ${ }^{22}$ Yao et al found that the addition of endostar to conventional chemotherapy treatment could effectively attenuate the development of peritoneal carcinomatosis and extend survival with high safety and tolerance in gastric cancer from a retrospective study. ${ }^{23}$ After meta-analysis, An et al concluded that the chemotherapy regimen of endostar, vinorelbine, and cisplatin improved the prognosis of patients with advanced non-small cell lung cancer without increasing the risk of toxicity. ${ }^{24}$ Other studies have 
also shown that endostar with conventional chemotherapy is an effective, well-tolerated regimen in tumors of pediatric osteosarcoma, nasopharyngeal carcinoma, non-small cell lung cancer, colorectal cancer, gastrointestinal cancer, and esophageal cancer. ${ }^{25-30}$ Apatinib is a novel, oral, small molecule tyrosine-kinase inhibitor of VEGFR. VEGFR can contribute to pathological angiogenesis, leading to tumor growth and metastasis. ${ }^{31,32}$ Many clinical studies have shown that apatinib prolongs survival with manageable side effects in solid tumors, including non-small cell lung cancer, gastroesophageal cancer, breast cancer, and hepatocellular carcinoma. ${ }^{32-38}$ Wang et al reported two patients with advanced esophageal cancer who could not tolerate chemotherapy. Both had dyspnea symptoms. However, these symptoms were significantly relieved quickly when they took apatinib. At the same time, their diseases were controlled for a certain time, which may demonstrate that apatinib is effective for some patients with esophageal cancer. ${ }^{39}$ In view of the clinical effectiveness of endostar and apatinib and the vascular correlation of MCC, it may be an effective treatment to select antivascular drug therapy.

\section{Conclusion}

Based on this case report and literature reviews of MCC, we understand that $\mathrm{MCC}$ is a rare tumor with neuroendocrine properties. It can be transferred to local lymph nodes in the early stage, and even disseminated to distant places. In this case report, for the first time, we report that antiangiogenic therapy is an extremely effective treatment for MCC, especially for patients who cannot tolerate chemotherapy and radiotherapy. In future, more clinical studies are needed to explore more efficacious treatment for MCC, as well as its biological behavior at a deeper level.

\section{Abbreviations}

CK-H, cytokeratin (high molecular weight); CK-L, cytokeratin (low molecular weight); Syn, synaptophysin.

\section{Acknowledgments}

This study was supported by the National Natural Science Foundation of China (grant 81672896) and the National Key Research and Development Program: Key Technology of Palliative Care and Nursing for Cancer Patients (ZDZX2017ZL-01). The authors thank the patient and his family members for their participation in this study and for their agreement to the publication of the report.

\section{Author contributions}

WJ and JLX drafted the manuscript. JLX, RW, and TTW participated in collation of the clinical data. YQS and LKL critically revised the paper. All authors contributed toward data analysis, drafting and revising the article, gave final approval of the version to be published, and agree to be accountable for all aspects of the work.

\section{Disclosure}

The authors report no conflicts of interest in this work.

\section{References}

1. Tang CK, Toker C. Trabecular carcinoma of the skin: further clinicopathologic and ultrastructural study. Mt Sinai J Med. 1979;46(5):516-523.

2. Czapiewski P, Biernat W. Merkel cell carcinoma - recent advances in the biology, diagnostics and treatment. Int J Biochem Cell Biol. 2014;53: 536-546.

3. Krasagakis K, Tosca AD. Overview of Merkel cell carcinoma and recent advances in research. Int J Dermatol. 2003;42(9):669-676.

4. Andea AA, Coit DG, Amin B, Busam KJ. Merkel cell carcinoma: histologic features and prognosis. Cancer. 2008;113(9):2549-2558.

5. Smith VA, Madan OP, Lentsch EJ. Tumor location is an independent prognostic factor in head and neck Merkel cell carcinoma. Otolaryngol Head Neck Surg. 2012;146(3):403-408

6. Gould E, Albores-Saavedra J, Dubner B, Smith W, Payne CM. Eccrine and squamous differentiation in Merkel cell carcinoma. An immunohistochemical study. Am J Surg Pathol. 1988;12(10):768-772.

7. Feng H, Shuda M, Chang Y, Moore PS. Clonal integration of a polyomavirus in human Merkel cell carcinoma. Science. 2008;319(5866): 1096-1100.

8. Tadmor T, Liphshitz I, Aviv A, Landgren O, Barchana M, Polliack A. Increased incidence of chronic lymphocytic leukaemia and lymphomas in patients with Merkel cell carcinoma - a population based study of 335 cases with neuroendocrine skin tumour. Br J Haematol. 2012;157(4):457-462.

9. Miettinen M. Keratin 20: immunohistochemical marker for gastrointestinal, urothelial, and Merkel cell carcinomas. Mod Pathol. 1995;8(4): 384-388.

10. Metz KA, Jacob M, Schmidt U, Steuhl KP, Leder LD. Merkel cell carcinoma of the eyelid: histological and immunohistochemical features with special respect to differential diagnosis. Graefes Arch Clin Exp Ophthalmol. 1998;236(8):561-566.

11. Schadendorf D, Lebbé C, Zur Hausen A, et al. Merkel cell carcinoma: epidemiology, prognosis, therapy and unmet medical needs. Eur $J$ Cancer. 2017;71:53-69.

12. Lebbe C, Becker JC, Grob JJ, et al. Diagnosis and treatment of Merkel Cell Carcinoma. European consensus-based interdisciplinary guideline. Eur J Cancer. 2015;51(16):2396-2403.

13. Veness MJ. Merkel cell carcinoma: improved outcome with the addition of adjuvant therapy. J Clin Oncol. 2005;23(28):7235-7236.

14. Garneski KM, Nghiem P. Merkel cell carcinoma adjuvant therapy: current data support radiation but not chemotherapy. J Am Acad Dermatol. 2007;57(1):166-169.

15. Davis MP, Miller EM, Rau RC, Johnson OE, Naille RA, Crnkovich MJ. The use of VP16 and cisplatin in the treatment of Merkel cell carcinoma. J Dermatol Surg Oncol. 1990;16(3):276-278.

16. Sharma D, Flora G, Grunberg SM. Chemotherapy of metastatic Merkel cell carcinoma: case report and review of the literature. Am J Clin Oncol. 1991;14(2):166-169.

17. Tai PT, Yu E, Winquist E, et al. Chemotherapy in neuroendocrine/ Merkel cell carcinoma of the skin: case series and review of 204 cases. J Clin Oncol. 2000;18(12):2493-2499.

18. Terheyden P, Becker JC. New developments in the biology and the treatment of metastatic Merkel cell carcinoma. Curr Opin Oncol. 2017; 29(3):221-226.

19. Nghiem PT, Bhatia S, Lipson EJ, et al. PD-1 Blockade with Pembrolizumab in Advanced Merkel-Cell Carcinoma. $N$ Engl J Med. 2016; 374(26):2542-2552. 
20. Dixelius J, Larsson H, Sasaki T, et al. Endostatin-induced tyrosine kinase signaling through the Shb adaptor protein regulates endothelial cell apoptosis. Blood. 2000;95(11):3403-3411.

21. Ling Y, Yang Y, Lu N, et al. Endostar, a novel recombinant human endostatin, exerts antiangiogenic effect via blocking VEGF-induced tyrosine phosphorylation of KDR/Flk-1 of endothelial cells. Biochem Biophys Res Commun. 2007;361(1):79-84.

22. Chen J, Yao Q, Huang M, et al. A randomized Phase III trial of neoadjuvant recombinant human endostatin, docetaxel and epirubicin as first-line therapy for patients with breast cancer (CBCRT01). Int $J$ Cancer. 2018;142(10):2130-2138.

23. Yao J, Fan L, Peng C, et al. Clinical efficacy of endostar combined with chemotherapy in the treatment of peritoneal carcinomatosis in gastric cancer: results from a retrospective study. Oncotarget. 2017;8(41): $70788-70797$.

24. An J, Lv W, Endostar LW. Endostar (rh-endostatin) versus placebo in combination with vinorelbine plus cisplatin chemotherapy regimen in treatment of advanced non-small cell lung cancer: A meta-analysis. Thorac Cancer. 2018;9(5):606-612.

25. Jiang S, Wang G, Dong Y. Endostar combined with chemotherapy in a pediatric osteosarcoma with pulmonary metastasis and malignant pleural effusion: a case report. Medicine. 2017;96(51):e9077.

26. Jin T, Jiang F, Jin QF, Piao YF, Chen XZ. Endostar Combined with Gemcitabine and Cisplatin Chemotherapy for Patients with Metastatic Nasopharyngeal Carcinoma: an Update. Transl Oncol. 2018;11(2): 286-291.

27. Cao D, Ge W, Wang H, Zhang L, Zheng Y, Zhang J. [Efficacy and safety of rh-endostatin combined with chemotherapy versus chemotherapy alone for advanced NSCLC: a meta-analysis review]. Zhongguo Fei Ai Za Zhi. 2011;14(5):404-413. Chinese.

28. Xu HX, Huang XE, Qian ZY, Xu X, Li Y, Li CGHXX, Cg L. Clinical observation of Endostar combined with chemotherapy in advanced colorectal cancer patients. Asian Pac J Cancer Prev. 2011;12(11):3087-3090.

29. Gao SR, Li LM, Xia HP, Wang GM, Xu HY, Wang AR. Clinical observation on recombinant human endostatin combined with chemotherapy for advanced gastrointestinal cancer. Asian Pac J Cancer Prev. 2015; 16(9):4037-4040
30. Deng WY, Song T, Li N, Luo SX, Li X. Clinical observation and therapeutic evaluation of $\mathrm{Rh}$-endostatin combined with DP regimen in treating patients with advanced esophageal cancer. Asian Pac J Cancer Prev. 2014;15(16):6565-6570.

31. Shibuya M. Tyrosine kinase receptor Flt/VEGFR family: its characterization related to angiogenesis and cancer. Genes Cancer. 2010;1(11): 1119-1123.

32. Li J, Zhao X, Chen L, et al. Safety and pharmacokinetics of novel selective vascular endothelial growth factor receptor-2 inhibitor YN968D1 in patients with advanced malignancies. BMC Cancer. 2010;10(1):529.

33. Tian S, Quan H, Xie C, et al. YN968D1 is a novel and selective inhibitor of vascular endothelial growth factor receptor-2 tyrosine kinase with potent activity in vitro and in vivo. Cancer Sci. 2011;102(7):1374-1380.

34. Li J, Qin S, Xu J, et al. Randomized, Double-Blind, Placebo-Controlled Phase III Trial of Apatinib in Patients With Chemotherapy-Refractory Advanced or Metastatic Adenocarcinoma of the Stomach or Gastroesophageal Junction. J Clin Oncol. 2016;34(13):1448-1454.

35. Li J, Qin S, Xu J, et al. Apatinib for chemotherapy-refractory advanced metastatic gastric cancer: results from a randomized, placebo-controlled, parallel-arm, phase II trial. J Clin Oncol. 2013;31(26):3219-3225.

36. Zhang L, Shi M, Huang C, et al. A phase II, multicenter, placebocontrolled trial of apatinib in patients with advanced nonsquamous non-small cell lung cancer (NSCLC) after two previous treatment regimens. J Clin Oncol. 2012;30(15 Suppl):7548.

37. Hu X, Zhang J, Xu B, et al. Multicenter phase II study of apatinib, a novel VEGFR inhibitor in heavily pretreated patients with metastatic triple-negative breast cancer. Int J Cancer. 2014;135(8):1961-1969.

38. Qin S. Apatinib in Chinese patients with advanced hepatocellular carcinoma: a phase II randomized, open-label trial. Asco Meet Abstr. 2014;32(15 Suppl):4019.

39. Wang W, Zhang L, Xie Y, Zhen T, Su G, Zang Q. Fatal hemoptysis in patients with advanced esophageal cancer treated with apatinib. Onco Targets Ther. 2018;11:2565-2570.
OncoTargets and Therapy

\section{Publish your work in this journal}

OncoTargets and Therapy is an international, peer-reviewed, open access journal focusing on the pathological basis of all cancers, potential targets for therapy and treatment protocols employed to improve the management of cancer patients. The journal also focuses on the impact of management programs and new therapeutic agents and protocols on

\section{Dovepress}

patient perspectives such as quality of life, adherence and satisfaction The manuscript management system is completely online and includes a very quick and fair peer-review system, which is all easy to use. Visit http://www.dovepress.com/testimonials.php to read real quotes from published authors. 